\title{
Fischer decompositions of kernels of Hermitean Dirac operators
}

\author{
F. Brackx*, H. De Schepper*, R. Lávička ${ }^{\dagger}$ and V. Souček ${ }^{\dagger}$ \\ ${ }^{*}$ Clifford Research Group, Faculty of Engineering, Ghent University \\ Building S22, Galglaan 2, B-9000 Gent, Belgium \\ ${ }^{\dagger}$ Mathematical Institute, Faculty of Mathematics and Physics, Charles University, \\ Sokolovská 83, 18675 Praha, Czech Republic
}

\begin{abstract}
In this note we describe explicitly irreducible decompositions of kernels of the Hermitean Dirac Operators. In [3], it is shown that these decompositions are essential for a construction of orthogonal (or even Gelfand-Tsetlin) bases of homogeneous Hermitean monogenic polynomials.
\end{abstract}

Keywords: Hermitean Clifford analysis, Fischer decomposition

PACS: (MSC 2000) 30G35

\section{PRELIMINARIES OF HERMITEAN CLIFFORD ANALYSIS}

Let $\left(e_{1}, \ldots, e_{m}\right)$ be an orthonormal basis of Euclidean space $\mathbb{R}^{m}$ and let $\mathbb{C}_{m}$ stand for the complex Clifford algebra constructed over $\mathbb{R}^{m}$ such that

$$
e_{\alpha} e_{\beta}+e_{\beta} e_{\alpha}=-2 \delta_{\alpha \beta}, \alpha, \beta=1, \ldots, m \text {. }
$$

As a basis for $\mathbb{C}_{m}$ one takes for any set $A=\left\{j_{1}, \ldots, j_{h}\right\} \subset\{1, \ldots, m\}$ the element $e_{A}=e_{j_{1}} \ldots e_{j_{h}}$, with $1 \leq j_{1}<j_{2}<\cdots<j_{h} \leq m$, together with $e_{\emptyset}=1$, the identity element. Euclidean space $\mathbb{R}^{m}$ is embedded in $\mathbb{C}_{m}$ by identifying $\left(X_{1}, \ldots, X_{m}\right)$ with the Clifford vector $\underline{X}=\sum_{\alpha=1}^{m} e_{\alpha} X_{\alpha}$, for which it holds that $\underline{X}^{2}=-|\underline{X}|^{2}$. Any Clifford number $a$ in $\mathbb{C}_{m}$ may thus be written as $a=\sum_{A} e_{A} a_{A}, a_{A} \in \mathbb{C}$ and its conjugation is defined by $a^{\dagger}=\sum_{A} \bar{e}_{A} a_{A}^{c}$, where the bar notation stands for the main anti-involution for which $\bar{e}_{\alpha}=-e_{\alpha}$, and $a_{A}^{c}$ denotes the complex conjugation of $a_{A}$. The Fischer dual of $\underline{X}$ is the vector valued Dirac operator $\partial_{\underline{X}}=\sum_{\alpha=1}^{m} e_{\alpha} \partial_{X_{\alpha}}$, underlying the notion of monogenicity of a function, the higher dimensional counterpart of holomorphy in the complex plane. More explicitly, a function $f$ defined and continuously differentiable in an open region $\Omega$ of $\mathbb{R}^{m}$ and taking values in the Clifford algebra $\mathbb{C}_{m}$ is called (left) monogenic in $\Omega$ if $\partial_{X}[f]=0$ in $\Omega$. As the Dirac operator factorizes the Laplacian: $\Delta_{m}=-\partial_{\underline{X}}^{2}$, monogenicity can be regarded as a refinement of harmonicity. As usual, inside the Clifford algebra $\mathbb{C}_{m}$ we can realize the Pin group $\operatorname{Pin}(m)$ as the set of finite products of unit vectors of $\mathbb{R}^{m}$ endowed with the Cliffford multiplication. The group $\operatorname{Pin}(m)$ is a double cover of the orthogonal group $O(m)$. For $\mathbb{C}_{m}$-valued functions $f(\underline{X})$, the so-called $L$-action of $\operatorname{Pin}(m)$ is given by $[L(s)(f)](\underline{X})=s P\left(s^{-1} \underline{X} s\right), s \in \operatorname{Pin}(m)$. This is the framework for what is sometimes called Euclidean Clifford analysis. A standard reference is e.g. [5].

The transition to Hermitean Clifford analysis consists in adding a complex structure $J$ to the above Euclidean setting, i.e. an $\mathrm{SO}(m)$-element $J$ for which $J^{2}=\mathbf{- 1}$ (see e.g. [1] for details). Note that a complex structure can exist only in the even dimensional case $m=2 n$. In the sequel, the complex structure $J$ is chosen to act upon the generators $e_{1}, \ldots, e_{2 n}$ of $\mathbb{C}_{2 n}$ as $J\left[e_{j}\right]=-e_{n+j}$ and $J\left[e_{n+j}\right]=e_{j}, j=1, \ldots, n$. The projection operators $\frac{1}{2}(\mathbf{1} \pm i J)$ associated with $J$ produce the main objects of the Hermitean setting by acting upon the corresponding ones in the Euclidean framework. First the so-called Witt basis elements $\left(\mathfrak{f}_{j}, \mathfrak{f}_{j}^{\dagger}\right)_{j=1}^{n}$ for $\mathbb{C}_{2 n}$ are obtained:

$$
\begin{aligned}
& \mathfrak{f}_{j}=\frac{1}{2}(\mathbf{1}+i J)\left[e_{j}\right]=\frac{1}{2}\left(e_{j}-i e_{n+j}\right), \quad j=1, \ldots, n \\
& \mathfrak{f}_{j}^{\dagger}=-\frac{1}{2}(\mathbf{1}-i J)\left[e_{j}\right]=-\frac{1}{2}\left(e_{j}+i e_{n+j}\right), \quad j=1, \ldots, n
\end{aligned}
$$


They satisfy the respective Grassmann and duality identities

$$
\begin{aligned}
\mathfrak{f}_{j} \mathfrak{f}_{k}+\mathfrak{f}_{k} \mathfrak{f}_{j} & =\mathfrak{f}_{j}^{\dagger} \mathfrak{f}_{k}^{\dagger}+\mathfrak{f}_{k}^{\dagger} \mathfrak{f}_{j}^{\dagger}=0, \quad j, k=1, \ldots, n \\
\mathfrak{f}_{j} \mathfrak{f}_{k}^{\dagger}+\mathfrak{f}_{k}^{\dagger} \mathfrak{f}_{j} & =\delta_{j k}, \quad j, k=1, \ldots, n
\end{aligned}
$$

whence they are isotropic. Next, a vector in $\mathbb{R}^{2 n}$ is now alternatively denoted by $\left(x_{1}, \ldots, x_{n}, y_{1}, \ldots, y_{n}\right)$ and identified with the Clifford vector $\underline{X}=\sum_{j=1}^{n}\left(e_{j} x_{j}+e_{n+j} y_{j}\right)$, producing the Hermitean Clifford variables $\underline{z}$ and $\underline{z}^{\dagger}$ :

$$
\underline{z}=\frac{1}{2}(\mathbf{1}+i J)[\underline{X}]=\sum_{j=1}^{n} \mathfrak{f}_{j} z_{j} \quad \text { and } \quad \underline{z}^{\dagger}=-\frac{1}{2}(\mathbf{1}-i J)[\underline{X}]=\sum_{j=1}^{n} \mathfrak{f}_{j}^{\dagger} z_{j}^{c}
$$

where complex variables $z_{j}=x_{j}+i y_{j}$ have been introduced, with complex conjugates $z_{j}^{c}=x_{j}-i y_{j}, j=1, \ldots, n$. Finally, the Euclidean Dirac operator $\partial_{\underline{X}}$ gives rise to the Hermitean Dirac operators $\partial_{\underline{z}}$ and $\partial_{\underline{z}}^{\dagger}$ :

$$
\partial_{\underline{\underline{z}}}^{\dagger}=\frac{1}{4}(\mathbf{1}+i J)\left[\partial_{\underline{X}}\right]=\sum_{j=1}^{n} \mathfrak{f}_{j} \partial_{z_{j}^{c}} \quad \text { and } \quad \partial_{\underline{z}}=-\frac{1}{4}(\mathbf{1}-i J)\left[\partial_{\underline{X}}\right]=\sum_{j=1}^{n} \mathfrak{f}_{j}^{\dagger} \partial_{z_{j}}
$$

involving the Cauchy-Riemann operators $\partial_{z_{j}^{c}}=\frac{1}{2}\left(\partial_{x_{j}}+i \partial_{y_{j}}\right)$ and their complex conjugates $\partial_{z_{j}}=\frac{1}{2}\left(\partial_{x_{j}}-i \partial_{y_{j}}\right)$ in the $z_{j}$-planes, $j=1, \ldots, n$. The Hermitean vector variables and Dirac operators are isotropic, i.e. $z^{2}=\left(z^{\dagger}\right)^{2}=0$ and $\partial_{\underline{z}}^{2}=\left(\partial_{\underline{z}}^{\dagger}\right)^{2}=0$, whence the Laplacian allows for the decomposition

$$
\Delta_{2 n}=4\left(\partial_{\underline{z}} \partial_{\underline{z}}^{\dagger}+\partial_{\underline{z}}^{\dagger} \partial_{\underline{z}}\right)=4\left(\partial_{\underline{z}}+\partial_{\underline{z}}^{\dagger}\right)^{2}
$$

while also

$$
\left(\underline{z}+\underline{z}^{\dagger}\right)^{2}=\underline{z} \underline{z}^{\dagger}+\underline{z^{\dagger}} \underline{z}=|\underline{z}|^{2}=\left|\underline{z}^{\dagger}\right|^{2}=|\underline{X}|^{2}
$$

Finally, a continuously differentiable function $g$ in an open region $\Omega$ of $\mathbb{R}^{2 n}$ with values in the complex Clifford algebra $\mathbb{C}_{2 n}$ is called (left) Hermitean monogenic (or h-monogenic) in $\Omega$ if and only if it satisfies in $\Omega$ the system $\partial_{\underline{z}} g=0=\partial_{\underline{z}}^{\dagger} g$. As $\partial_{\underline{X}}=2\left(\partial_{\underline{z}}^{\dagger}-\partial_{\underline{z}}\right)$ h-monogenicity can be regarded as a refinement of monogenicity.

In comparison with the Euclidean setting the symmetry in the Hermitean framework is given not by the whole group Pin $(2 n)$ but only by its subgroup $\operatorname{Pin}_{J}(2 n)$. The subgroup $\operatorname{Pin}_{J}(2 n)$ consists of elements of the group $\operatorname{Pin}(2 n)$ commuting with the element $s_{J}$ which corresponds to the complex structure $J$ under the double cover of $O(2 n)$ by $\operatorname{Pin}(2 n)$. Moreover, the group $\operatorname{Pin}_{J}(2 n)$ is a double cover of the group $O_{J}(2 n)$, the subgroup of $O(2 n)$ containing just elements which commute with the complex structure $J$. Let us note that the group $S O_{J}(2 n)$ defined analogously can be seen as a realization of the unitary group $U(n)$.

In what follows, we consider spinor valued functions. Spinor space $\mathbb{S}$ is realized within the Clifford algebra $\mathbb{C}_{2 n}$ as $\mathbb{S}=\mathbb{C}_{2 n} I \cong \mathbb{C}_{n} I$ where $I$ is a suitable primitive idempotent, say $I=I_{1} \ldots I_{n}$ with $I_{j}=\mathfrak{f}_{j} f_{j}^{\dagger}, j=1, \ldots, n$. As $\mathfrak{f}_{j} I=0$, $j=1, \ldots, n$, we also have that $\mathbb{S} \cong \bigwedge_{n}^{* \dagger} I$ where $\bigwedge_{n}^{* \dagger}$ stands for the complex Grassmann algebra generated by $\left\{\mathrm{f}_{1}^{\dagger}, \ldots, \mathrm{f}_{n}^{\dagger}\right\}$. Hence spinor space $\mathbb{S}$ decomposes further into homogeneous parts as

$$
\mathbb{S}=\bigoplus_{r=0}^{n} \mathbb{S}^{(r)} \quad \text { with } \mathbb{S}^{(r)}=\left(\bigwedge_{n}^{\dagger}\right)^{(r)} I=\operatorname{span}_{\mathbb{C}}\left(\mathfrak{f}_{k_{1}}^{\dagger} \mathfrak{f}_{k_{2}}^{\dagger} \cdots \mathrm{f}_{k_{r}}^{\dagger}:\left\{k_{1}, \ldots, k_{r}\right\} \subset\{1, \ldots, n\}\right)
$$

\section{FISCHER DECOMPOSITIONS}

Recently in [1], an analogue of the Fischer decomposition for homogeneous polynomials has been obtained also in the setting of Hermitean Clifford analysis. From the point of view of representation theory, the Fischer decomposition is nothing else than an irreducible decomposition of a given invariant space of polynomials. More explicitly, let us denote by $\mathscr{P}_{a, b}^{r}$ the space of $\mathbb{S}^{(r)}$-valued polynomials $p$ in $\mathbb{R}^{2 n}$ which are $a$-homogeneous in the variables $z_{j}$ and at the same time $b$-homogeneous in the variables $z_{j}^{c}$, that is,

$$
p\left(\lambda z_{1}, \ldots, \lambda z_{n}, \mu z_{1}^{c}, \ldots, \mu z_{n}^{c}\right)=\lambda^{a} \mu^{b} p\left(z_{1}, \ldots, z_{n}, z_{1}^{c}, \ldots, z_{n}^{c}\right) .
$$


Moreover, let $\mathscr{M}_{a, b}^{r}$ stand for the space of polynomials of $\mathscr{P}_{a, b}^{r}$ which are Hermitean monogenic. Then, under the action of the group $\operatorname{Pin}_{J}(2 n)$, the space $\mathscr{P}_{a, b}^{r}$ has the following irreducible (not multiplicity free) decomposition (see [1, Proposition 1] or [4]):

$$
\begin{gathered}
\mathscr{P}_{a, b}^{r}=\mathscr{M}_{a, b}^{r} \oplus \bigoplus_{j=0}^{\min (a, b-1)}|\underline{z}|^{2 j} \underline{z}^{\dagger} \mathscr{M}_{a-j, b-j-1}^{r-1} \oplus \bigoplus_{j=0}^{\min (a-1, b)}|\underline{z}|^{2 j} \underline{z} \mathscr{M}_{a-j-1, b-j}^{r+1} \oplus \\
\oplus \bigoplus_{j=0}^{\min (a-1, b-1)}\left(\underline{z}^{\dagger} \underline{z}\right)^{j+1} \mathscr{M}_{a-j-1, b-j-1}^{r} \oplus \bigoplus_{j=0}^{\min (a-1, b-1)}\left(\underline{z} \underline{z}^{\dagger}\right)^{j+1} \mathscr{M}_{a-j-1, b-j-1}^{r} .
\end{gathered}
$$

The main aim of this note is to describe Fischer decompositions which are essential for a construction of orthogonal (or even Gelfand-Tsetlin) bases of homogeneous Hermitean monogenic polynomials. In [3], it is explained that for the construction it is important to know irreducible decompositions of the spaces

$$
\operatorname{Ker}_{a, b}^{r} \partial_{\underline{z}}=\left\{p \in \mathscr{P}_{a, b}^{r} \mid \partial_{\underline{z}} p=0\right\} \text { and } \operatorname{Ker}_{a, b}^{r} \partial_{\underline{z}}^{\dagger}=\left\{p \in \mathscr{P}_{a, b}^{r} \mid \partial_{\underline{z}}^{\dagger} p=0\right\} .
$$

Now we obtain such decompositions.

Theorem 1. Let $1 \leq r \leq n-1$. Then the following statements hold:

(i) Under the action of $\operatorname{Pin}_{J}(2 n)$, the space $\operatorname{Ker}_{a, b}^{r} \partial_{\underline{z}}$ has the multiplicity free irreducible decomposition

$$
\operatorname{Ker}_{a, b}^{r} \partial_{\underline{z}}=\mathscr{M}_{a, b}^{r} \oplus \bigoplus_{j=0}^{\min (a, b-1)}|\underline{z}|^{2 j} \underline{z}^{\dagger} \mathscr{M}_{a-j, b-j-1}^{r-1} \oplus \bigoplus_{j=0}^{\min (a-1, b-1)}|\underline{z}|^{2 j}\left(\underline{z} \underline{z}^{\dagger} \underline{\underline{z}}+\frac{(a-j-1+r)}{(a+r)} \underline{z} \underline{z}^{\dagger}\right) \mathscr{M}_{a-j-1, b-j-1}^{r} .
$$

(ii) Under the action of $\operatorname{Pin}_{J}(2 n)$, the space $\operatorname{Ker}_{a, b}^{r} \partial_{\underline{z}}^{\dagger}$ has the multiplicity free irreducible decomposition

$$
\operatorname{Ker}_{a, b}^{r} \partial_{\underline{z}}^{\dagger}=\mathscr{M}_{a, b}^{r} \oplus \bigoplus_{j=0}^{\min (a-1, b)}|\underline{z}|^{2 j} \underline{z} \mathscr{M}_{a-j-1, b-j}^{r+1} \oplus \bigoplus_{j=0}^{\min (a-1, b-1)}|\underline{z}|^{2 j}\left(\underline{z z}{ }^{\dagger}+\frac{(b-j-1+n-r)}{(b+n-r)} \underline{z} \underline{z}^{\dagger}\right) \mathscr{M}_{a-j-1, b-j-1}^{r} .
$$

Remark 1. It is easy to see that $\operatorname{Ker}_{a, b}^{0} \partial_{\underline{z}}=\mathscr{M}_{a, b}^{0}, \operatorname{Ker}_{a, b}^{n} \partial_{\underline{z}}=\mathscr{P}_{a, b}^{n}, \operatorname{Ker}_{a, b}^{0} \partial_{\underline{z}}^{\dagger}=\mathscr{P}_{a, b}^{0}$ and $\operatorname{Ker}_{a, b}^{n} \partial_{\underline{z}}^{\dagger}=\mathscr{M}_{a, b}^{n}$.

In order to prove Theorem 1 we need some lemmas. But first define the following Euler operators:

$$
E_{z}=\sum_{j=1}^{n} z_{j} \partial_{z_{j}}, \quad E_{z^{c}}=\sum_{j=1}^{n} z_{j}^{c} \partial_{z_{j}^{c}} \text { and } \beta=\sum_{j=1}^{n} \mathfrak{f}_{j}^{\dagger} \mathfrak{f}_{j}
$$

For $p \in \mathscr{P}_{a, b}^{r}$, it is easy to see that $E_{z} p=a p, E_{z^{c}} p=b p$ and $\beta p=r p$. See [1] for details. Putting $A=E_{z}+\beta$ and $B=E_{z^{c}}+n-\beta$, we have thus that, for each $p \in \mathscr{P}_{a, b}^{r}, A p=(a+r) p$ and $B p=(b+n-r) p$. Moreover, we have that

$$
\left\{\underline{z}, \partial_{\underline{z}}\right\}=A, \quad\left\{\underline{z}^{\dagger}, \partial_{\underline{z}}^{\dagger}\right\}=B \text { and }\left\{\underline{z}, \partial_{\underline{z}}^{\dagger}\right\}=0=\left\{\underline{z}^{\dagger}, \partial_{\underline{z}}\right\}
$$

where $\{T, S\}=T S+S T$ (see e.g. [1]). Using these relations, it is easy to prove the following ones.

Lemma 1. We have that

$$
\begin{array}{lll}
{\left[\partial_{\underline{z}},|\underline{z}|^{2 j}\right]=j|\underline{z}|^{2(j-1)} \underline{z}^{\dagger},} & {\left[\partial_{\underline{z}},\left(\underline{z^{\dagger}} \underline{z}\right)^{j+1}\right]=-|\underline{z}|^{2 j} \underline{z}^{\dagger} A,} & {\left[\partial_{\underline{z}},\left(\underline{z z^{\dagger}}\right)^{j+1}\right]=|\underline{z}|^{2 j} \underline{z}^{\dagger}(A+j+1),} \\
{\left[\partial_{\underline{z}}^{\dagger},|\underline{z}|^{2 j}\right]=j|\underline{z}|^{2(j-1)} \underline{z},} & \left.\left[\partial_{\underline{z}}^{\dagger},(\underline{z z})^{\dagger}\right)^{j+1}\right]=-|\underline{z}|^{2 j} \underline{z} B, & {\left[\partial_{\underline{z}}^{\dagger},\left(\underline{z^{\dagger}} \underline{z}\right)^{j+1}\right]=|\underline{z}|^{2 j} \underline{z}(B+j+1)}
\end{array}
$$

where $[T, S]=T S-S T$.

Lemma 2. We have that

$$
\mathscr{P}_{a, b}^{r}=\operatorname{Ker}_{a, b}^{r} \partial_{\underline{z}} \oplus \underline{z} \operatorname{Ker}_{a-1, b}^{r+1} \partial_{\underline{z}}=\operatorname{Ker}_{a, b}^{r} \partial_{\underline{z}}^{\dagger} \oplus \underline{z}^{\dagger} \operatorname{Ker}_{a, b-1}^{r-1} \partial_{\underline{z}}^{\dagger} .
$$

Moreover, the projections $P$ and $P^{\dagger}$ of the space $\mathscr{P}_{a, b}^{r}$ onto the spaces $\operatorname{Ker}_{a, b}^{r} \partial_{\underline{z}}$ and $\operatorname{Ker}_{a, b}^{r} \partial_{\underline{z}}^{\dagger}$ are given by $P=\partial_{\underline{z}} \underline{z} A^{-1}$ and $P^{\dagger}=\partial_{\underline{z}}^{\dagger} \underline{z}^{\dagger} B^{-1}$, respectively. 
Proof. We may refer to [2], but it is also clear from the relations (3).

Proof of Theorem 1. Applying the projections $P$ and $P^{\dagger}$ of Lemma 2 to the Fischer decomposition (2), we easily get the required decompositions of the spaces $\operatorname{Ker}_{a, b}^{r} \partial_{\underline{z}}$ and $\operatorname{Ker}_{a, b}^{r} \partial_{\underline{z}}^{\dagger}$. Indeed, using the relations (3) and Lemma 1, we have that

$$
P\left(\mathscr{P}_{a, b}^{r}\right)=\operatorname{Ker}_{a, b}^{r} \partial_{\underline{z}}, \quad P\left(\mathscr{M}_{a, b}^{r}\right)=\mathscr{M}_{a, b}^{r}, \quad P\left(|\underline{z}|^{2 j} \underline{z}^{\dagger} \mathscr{M}_{a-j, b-j-1}^{r-1}\right)=|\underline{z}|^{2 j} \underline{z}^{\dagger} \mathscr{M}_{a-j, b-j-1}^{r-1}
$$

and

$$
P\left(\left(\underline{z}^{\dagger} \underline{z}\right)^{j+1} \mathscr{M}_{a-j-1, b-j-1}^{r}\right)=|\underline{z}|^{2 j}\left(\underline{z}^{\dagger} \underline{z}+\frac{(a-j-1+r)}{(a+r)} \underline{z} \underline{z}^{\dagger}\right) \mathscr{M}_{a-j-1, b-j-1}^{r}
$$

Moreover, the operator $P$ vanishes on the remaining pieces $|\underline{z}|^{2 j} \underline{z} \mathscr{M}_{a-j-1, b-j}^{r+1}$ and $\left(\underline{z} \underline{z}^{\dagger}\right)^{j+1} \mathscr{M}_{a-j-1, b-j-1}^{r}$. Similar computations are, of course, valid for the operator $P^{\dagger}$, which completes the proof.

\section{ACKNOWLEDGMENTS}

R. Lávička and V. Souček acknowledge support by the institutional grant MSM 0021620839 and by grant GA CR 201/08/0397.

\section{REFERENCES}

1. F. Brackx, H. De Schepper, D. Eelbode, V. Souček, The Howe Dual Pair in Hermitean Clifford Analysis, Rev. Mat. Iberoamericana $26(2010)(2), 449-479$.

2. F. Brackx, H. De Schepper, R. Lávička, V. Souček, The Cauchy-Kovalevskaya Extension Theorem in Hermitean Clifford Analysis (submitted).

3. F. Brackx, H. De Schepper, R. Lávička, V. Souček, Gelfand-Tsetlin Bases of Orthogonal Polynomials in Hermitean Clifford Analysis (to appear).

4. F. Brackx, H. De Schepper, V. Souček, Fischer Decompositions in Euclidean and Hermitean Clifford Analysis (to appear).

5. R. Delanghe, F. Sommen, V. Souček, Clifford algebra and spinor-valued functions - A function theory for the Dirac operator, Kluwer Academic Publishers (Dordrecht, 1992). 